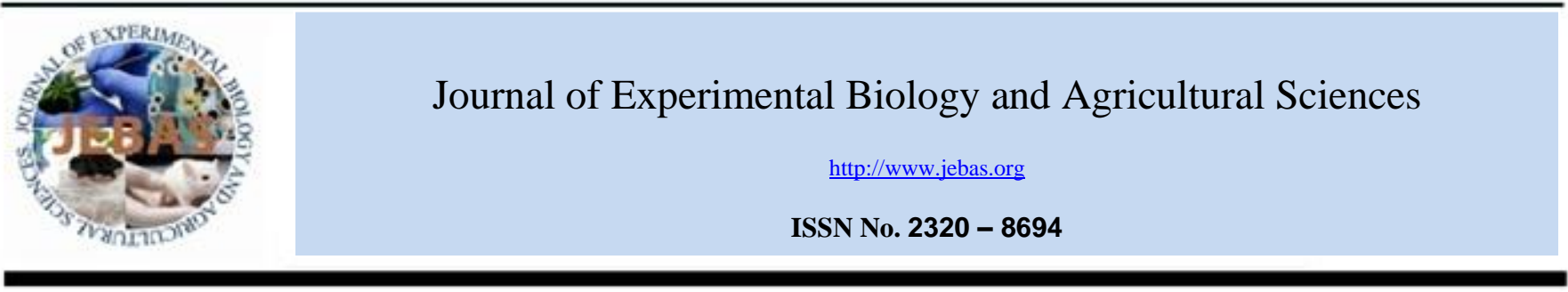

\title{
PARASITOLOGICAL, BIOCHEMICAL AND CLINICAL OBSERVATIONS IN PONIES EXPERIMENTALLY INFECTED WITH Trypanosoma evansi
}

\section{Yadav SC ${ }^{1, *}$, Jaideep Kumar ${ }^{2}$, Gupta $\mathrm{AK}^{1}$, Jerome A ${ }^{4}$, Prabhat Kumar ${ }^{3}$, Rajender Kumar ${ }^{1}$, Kanika Tehri $^{3}$ and Ritesh Kumar ${ }^{3}$}

${ }^{1}$ ICAR-National Research Centre on Equines, Sirsa Road, Hisar, India

${ }^{2}$ Research Scholar, Guru Jambheshwar University of Science and Technology, Hisar, India

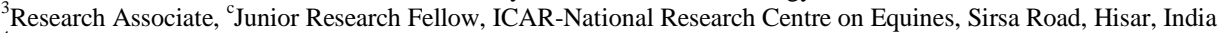

${ }^{4}$ ICAR-Central Institute for Research on Buffaloes, Sirsa Road, Hisar, India

Received - October 25, 2016; Revision - November 08, 2016; Accepted - November 25, 2016

Available Online - December 04, 2016

DOI: http://dx.doi.org/10.18006/2016.4(Spl-4-EHIDZ).S144.S150

KEYWORDS
Trypanosoma evansi
Ponies
Surra
Biochemical changes
Clinical signs
Haematology
Parasitaemia

\begin{abstract}
The present investigation aimed to study the parasitological, biochemical and clinical alterations in ponies during the course of Trypanosoma evansi experimental infection. Six female ponies were experimentally infected sub-cutaneously with mice adapted $2 \times 10^{6} \mathrm{~T}$. evansi parasites, isolated from naturally infected horse, while two ponies were maintained as uninfected healthy controls. All six ponies became parasitologically positive between 5-7 days post infection (DPI) tested by standard parasitological detection method (SPDM) by blood smear examination showing varying degree of parasitaemia and two prominent peaks during the course of infection. The main clinical signs observed were intermittent fever, weakness, emaciation, anaemia, anorexia and incoordination in hind quarters leading to significant weight loss at terminal stage of infection. All the infected ponies developed subacute to acute disease within 56 days and reached to recumbency stage. Of them, four ponies died at different stages of infection and few of them showing neurological signs at terminal stage of infection. The present investigation also revealed that horse ponies are more susceptible than donkeys in experimental infection of $T$. evansi. Haematological studies showed a gradual fall in the levels of haemoglobin $(\mathrm{Hb})$, hematocrit (HCT) and red blood cell (RBC) count from 10.57 to $4.83(\mathrm{~g} / \mathrm{dl}), 32.81$ to $16.33(\%)$ and 8.53 to $3.33\left(\times 10^{12}\right.$ cells/l) respectively, in infected animals over the study period. Serum
\end{abstract}

* Corresponding author

E-mail: yadavsc@ rediffmail com (S. C. Yadav)

Peer review under responsibility of Journal of Experimental Biology and Agricultural Sciences.

Production and Hosting by Horizon Publisher India [HPI] (http://www.horizonpublisherindia.in/).

All rights reserved.
All the article published by Journal of Experimental Biology and Agricultural Sciences is licensed under a Creative Commons Attribution-NonCommercial 4.0 International License Based on a work at www.jebas.org.

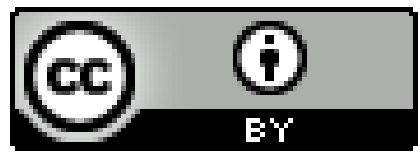


urea, uric acid, triglyceride, cholesterol, bilirubin indirect (BID) and total bilirubin (BIT) contents increased, while albumin contents significantly decreased in $T$. evansi infected ponies at different stages indicating impairment of liver and kidney functions. However, no changes in parasitological and biochemical responses were observed in the healthy controls.

\section{Introduction}

Animal trypanosomosis is caused by different species of trypanosomes, including Trypanosoma vivax, T. evansi, $T$. congolense, T. simiae and T. equiperdum in different parts of the world. Of them, $T$. evansi is the most widely spread organism with the greatest range of hosts (Hoare, 1972), thus making it one of the most significant animal health problems in the world. T. evansi is transmitted mechanically and noncyclically by haematophagus flies such as horseflies (Tabanus) and stable flies (Stomoxys) which act both as vector and host reservoir. This parasite is responsible for the disease 'surra' in domestic as well as wild animals, causing severe constraints to agriculture development and leading to significant impact on livestock in Asia, sub-Saharan Africa, and Latin America. In India, trypanosomosis caused by $T$. evansi is enzootic, since it affects many species of domestic and wild animals. Several sporadic outbreaks of equine trypanosomosis have been reported from different states (Yadav et al., 2012; Kumar et al., 2013). The course of the disease lasts from one week to six months (Woo, 1977) and usually results in emaciation and death. The pre-patent period varies from 4 to 13 days and parasitaemia displays an undulating course (Ramirez et al., 1979).

Anaemia is commonly found in horses, donkeys, dogs and coatis experimentally infected with $T$. evansi (Soodan et al., 1996; Marques et al., 2000; Aquino et al., 2002; Herrera et al., 2002). The disease manifests itself in different forms: acute, sub-acute, chronic and in-apparent. Clinical signs are only indicative of surra, which include progressive weakness, emaciation, fever, anaemia and death of affected animal. More recently rising trends of neurological cases due to T. evansi have also been reported, showing marked ataxia, hyperexcitability, circling, depression, gradual onset of paralysis of hind quarters (Rodrigues et al., 2009; Berlin et al., 2009; Ranjith kumar et al., 2013). The disease progresses in two phases; an acute phase, characterized by high levels of parasitaemia and noticeable clinical symptoms, and a chronic phase, characterized by low parasitaemia, which can either lead to emaciation or become clinically unapparent with undetectable changes in variables such as body temperature and haematocrit count (Fernández et al., 2009). The chronic form is most common and is likely to present an association with secondary infection due to immune-suppression caused by T. evansi infection (Ahmed, 2008).

Some alterations in blood biochemistry, including decrease in blood albumin and increase in globulin levels, hypoglycemia and increase in icterus index, have been reported in donkeys and horses (Soodan et al., 1996; Marques et al., 2000). The aim of the present study was to record the characteristic clinical course of the disease as well as parasitological, haematological and biochemical aspects in ponies experimentally infected with Indian isolate of T. evansi.

\section{Material and Methods}

\subsection{Source and maintenance of T. evansi}

T. evansi was isolated at National Research Centre on Equines at Hisar, Haryana during 2009 from infected horse and was maintained in mice by in vivo propagation. Briefly, the blood was collected from the parasitological positive horse in ethylene diamine tetra acetic acid (EDTA) and injected intraperitoneally (i/p) in mice. The level of parasitaemia was checked microscopically by collecting blood daily from tail of the mice. After attaining parasitaemic peak, T. evansi isolate was maintained in the laboratory by inoculating the $10^{4}$ parasites in naive mice, through $\mathrm{i} / \mathrm{p}$ route. The parasites were also kept in cryopreserved form in liquid nitrogen for further use, as and when required.

\subsection{Experimental infection in ponies}

Eight female ponies, aged 9-12 months, procured from local market, were divided in two groups (infected and healthy groups), comprising of six (P1 to P6) and two ponies (P7 \& P8), respectively. All the animals were examined for the presence of helminth ova/oocyst and treated with albendazol@10 mg/kg body weight six weeks prior to experiment. Further these animals were also confirmed for abesence of T. evansi and Theilaria equi antibodies prior to infection by antibody ELISA. These animals were housed in fly proof stable and maintained throughout the experiment under intensive system of management. They were fed on balanced diet consisting of water and green fodder ad libitum, during the experiment.

The experimental infection was set up in six ponies by inoculating $2 \times 10^{6}$ parasites sub-cutaneously (s/c) / pony. Another group of two ponies was kept as uninfected control during the course of experiment. The serum/blood samples were collected initially, at short intervals i.e. on day $0,3,5,7$, 10 and 14 from both groups. Thereafter, weekly blood samples were collected for parasitological, biochemical and clinical observations till 56 days post-infection. During the period, three ponies which became terminally ill reached in recumbent stage and were euthanized. The remaining three ponies at the end of experiment were treated with quinapyrimine sulphate @ $3 \mathrm{mg} / \mathrm{kg}$ body weight and monitored clinically after treatment. 
The animal experimentation was carried out according to the rules and regulations set forth by Committee for the Purpose of Control and Supervision of Experiments on Animals (CPCSEA) Animal Welfare Division, Ministry of Environment, Government of India. The research protocol for the experimentation was duly approved by the Institute Animal Ethics Committee of the National Research Centre on Equines, Hisar, Haryana.

\subsection{Parasitological observations}

During the course of experiment, the parasites were observed by wet blood smear examination and further counted in 50 microscopic fields at 400x magnification in thin blood smears stained with Giemsa (Cadioli et al., 2006).

2.4 Haematological and biochemical indices

Erythrocyte count, packed cell volume and haemoglobin content were obtained from HM5 Vet Scan haematology analyzer (Abaxis, Pvt. Ltd, USA) as per standard procedure using the Vet Scan haematology kit, while biochemical metabolites related to liver and kidney functions mainly [Urea, Triglyceride (Tgl), Cholesterol (Chol), Albumin (Alb), Total Serum Protein (Prot), Bilirubin indirect (BID) and Total bilirubin (BIT), Creatinine (Cre), Uric acid (UA), Calcium $(\mathrm{Cal})]$ were evaluated at different intervals in serum samples of both healthy and infected animals using XL system packs for each metabolite in a Clinical Chemistry Analyzer (ERBAEM200).

\subsection{Statistical Analysis}

Statistical analysis was performed using SPSS (version 16), using repeated measures ANOVA. Data represented as mean I $\mathrm{S} E$ and considered significant at $\mathrm{p}<0.05$

\section{Results}

\subsection{Parasitological observations}

All six ponies inoculated sub-cutaneously with $2 \times 10^{6}$ T. evansi became parasitologically positive by 5-7 DPI as tested by standard parasitological detection method (SPDM) using wet/ thin blood smear examination. Parasitaemia was consistent in all ponies (low to moderate) and parasites were regularly observed in blood examination during the course of experiment except $10^{\text {th }}$ and $35^{\text {th }}$ DPI (only P3 showed high count). The first peak of parasitaemia occurred on day 7 with an average of 416.17 parasites followed by next peak by 28 DPI with an average parasite count of 324.67 (Figure. 1). It was interesting to note that all the individual ponies on day 10 had nil parasite count except pony P-3 which got high parasite count at 35 DPI. Thereafter, all the ponies showed relapsing or undulating parasitaemia ranging from only few trypanosomes in blood to maximum densities 1-6 x10 7 parasites / ml. Body temperature increased up to $102-107^{\circ} \mathrm{F}$ but was intermittent in nature throughout the experiment. The first rise in temperature occurred on $5^{\text {th }}$ day after the appearance of trypanosomes in the blood, followed by a succession of peaks.

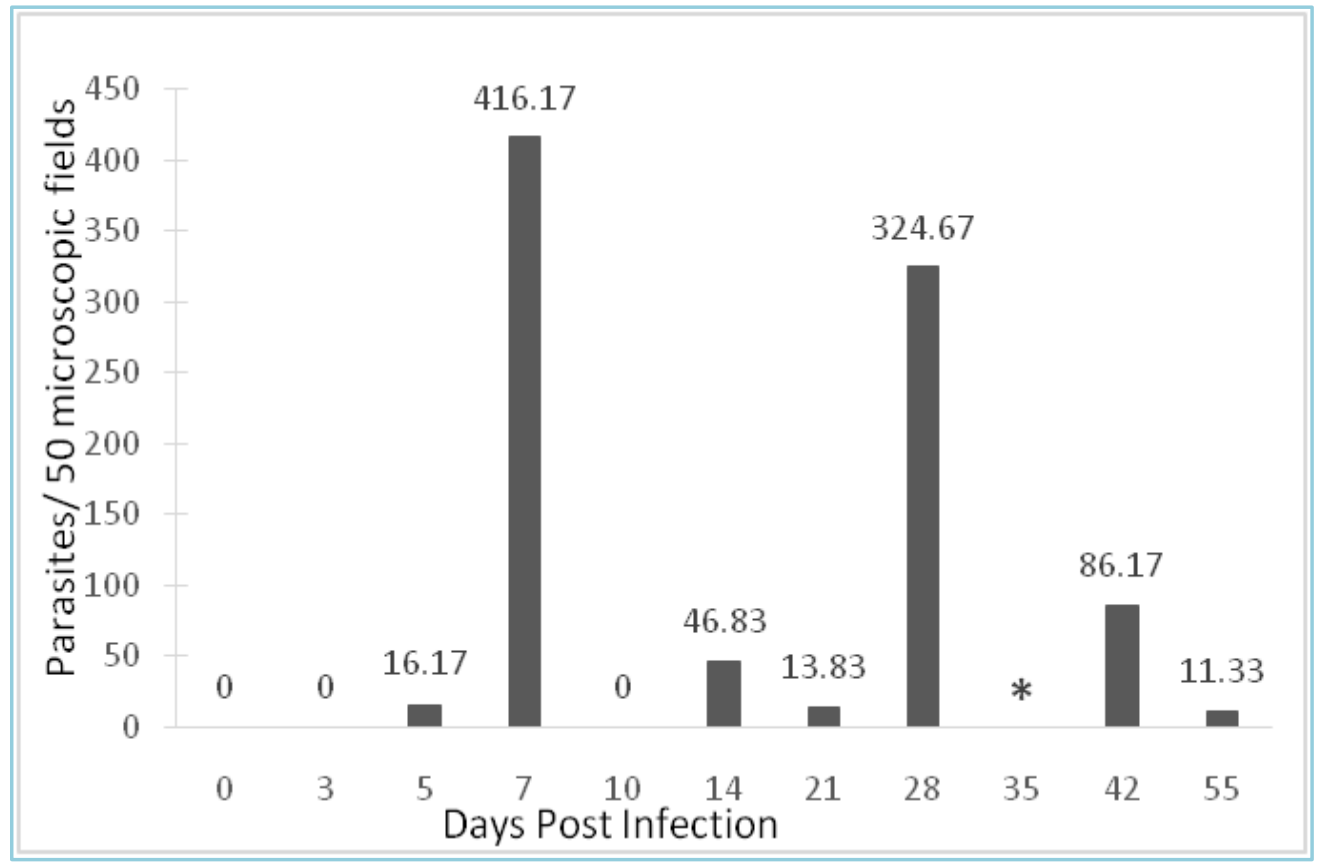

Figure 1 Parasite count in ponies infected with $T$. evansi at different intervals during infection.

*Only pony $\mathrm{P} 3$ was recorded with high parasite count of $439 *$ parasites at $35 \mathrm{DPI}$, while all other five infected ponies had nil count 

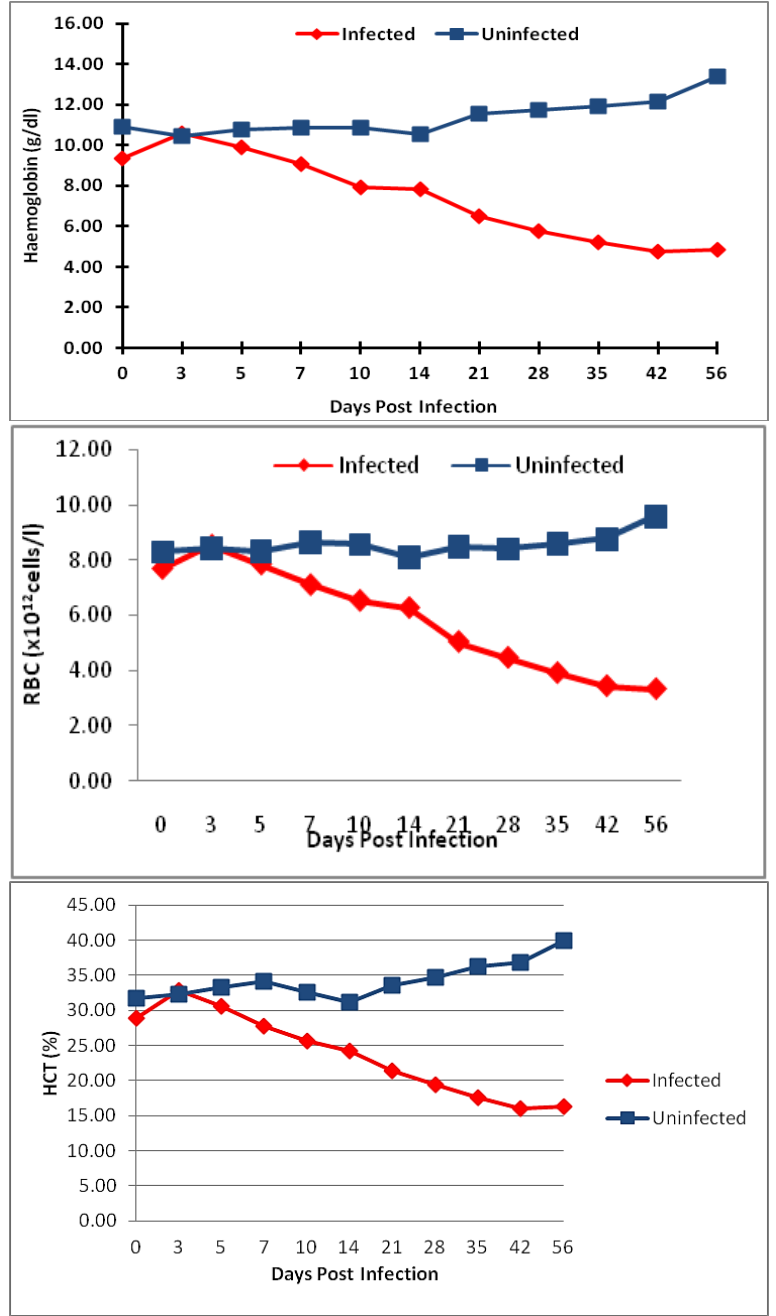

Figure 2 Changes in haemoglobin, RBC and hematocrit values in T. Evansi infected and uninfected ponies.

\subsection{Clinical signs}

Apart from increased body temperature, there were few more symptoms of disease during the first 4 weeks of infection. The main clinical signs observed in most of ponies were intermittent fever, fast breathing, lacrimation from eyes and became anorexic by 6 DPI. All the infected ponies displayed progressive emaciation, jaundice, mucosal pallor and in few cases sub-mandibular oedema also. Besides this, motor incoordination of hind limbs was observed (pony 4 and 5), reduced appetite and lateral recumbency (pony 1, $2 \& 5$ ) while ponies $3 \& 4$ showed oedema in brisket, abdominal regions, staggering gait and incoordination in hind quarters were also observed during the course of infection. At terminal stage of disease, all the ponies were reluctant to move, revealed pronounced hind quarters weakness with ataxia and incoordination of the hind limbs, demonstrating staggering gait with unsteady and irregular steps. Ponies subjected to physical exertion readily fell down and were unable to support weight on the hind limbs by 56 DPI.

The experiment was terminated at $61 \mathrm{DPI}$, as three ponies (P1, P2 \& P5) died/ euthanized during 58-61 DPI while rest three of the debilitated ponies, which showed sub-acute disease (unable to stand, walk and anorexic) were treated with quinapyrimine sulphate $(3 \mathrm{mg} / \mathrm{kg}$ body weight). After treatment, ponies were monitored for parasitaemia and found negative by $48 \mathrm{hrs}$ using SPDM methods. These animals recovered well at 10 weeks post-treatment, showing normal haematological indices and maintained good health, except for pony- 4 that showed acute neurological signs, tilting of head, circling motion, hyper-excitability and was finally recumbent and euthanized.

\subsection{Haematological observations}

Haematological studies showed that there was a continuous and sharp fall $(\mathrm{p}<0.05)$ in the levels of haemoglobin $(\mathrm{Hb})$, hematocrit (HCT) and red blood cell (RBC) count from 10.57 to $4.83(\mathrm{~g} / \mathrm{dl}), \quad 32.81$ to $16.33(\%)$ and 8.53 to 3.33 $\left(\times 10^{12}\right.$ cells/l), respectively in infected animals (Figure. 2) while in healthy ponies, no significant change in these parameters was observed. Moreover, no clinical, parasitological and biochemical changes were detected in two uninfected control ponies.

\subsection{Changes in biochemical indices}

Blood urea contents ranged from 20.52 to $57.37 \mathrm{mg} / \mathrm{dl}$ and 14.65 to $22.05 \mathrm{mg} / \mathrm{dl}$, while uric acid content ranged from 0.84 to 1.96 and 0.66 to $0.89 \mathrm{mg} / \mathrm{dl}$ in infected and healthy ponies, respectively (Figure 3). In infected ponies, urea content increased significantly 7 DPI onward and remained high throughout the experiment duration as compared to healthy ponies and zero days of infected ponies. Further a very sharp increase in this index was observed after 56 DPI in three ponies (P1, P2 and P5) which died on 61DPI. In rest of the three infected ponies (P3, P4 and P6), which survived on 61 DPI, urea content either remained static or decreased slowly. Uric acid content also showed a significant increase after 21 DPI and followed similar pattern between 56 to 61 DPI. No significant increase was observed in creatinine and calcium contents of infected ponies as compared to healthy ones. Triglyceride content showed a continuous increase in infected ponies 7 DPI. Further cholesterol, protein BID and BIT contents increased significantly about one to two weeks before death of infected ponies (P1, P2 and P5) while albumin content decreased sharply in later one as compared to healthy ponies. Among infected ponies, three ponies which remained alive, changes in levels of various metabolites were appreciably high between 7 to 35 DPI but during later stages (56 DPI onward), no significant increase was observed. 

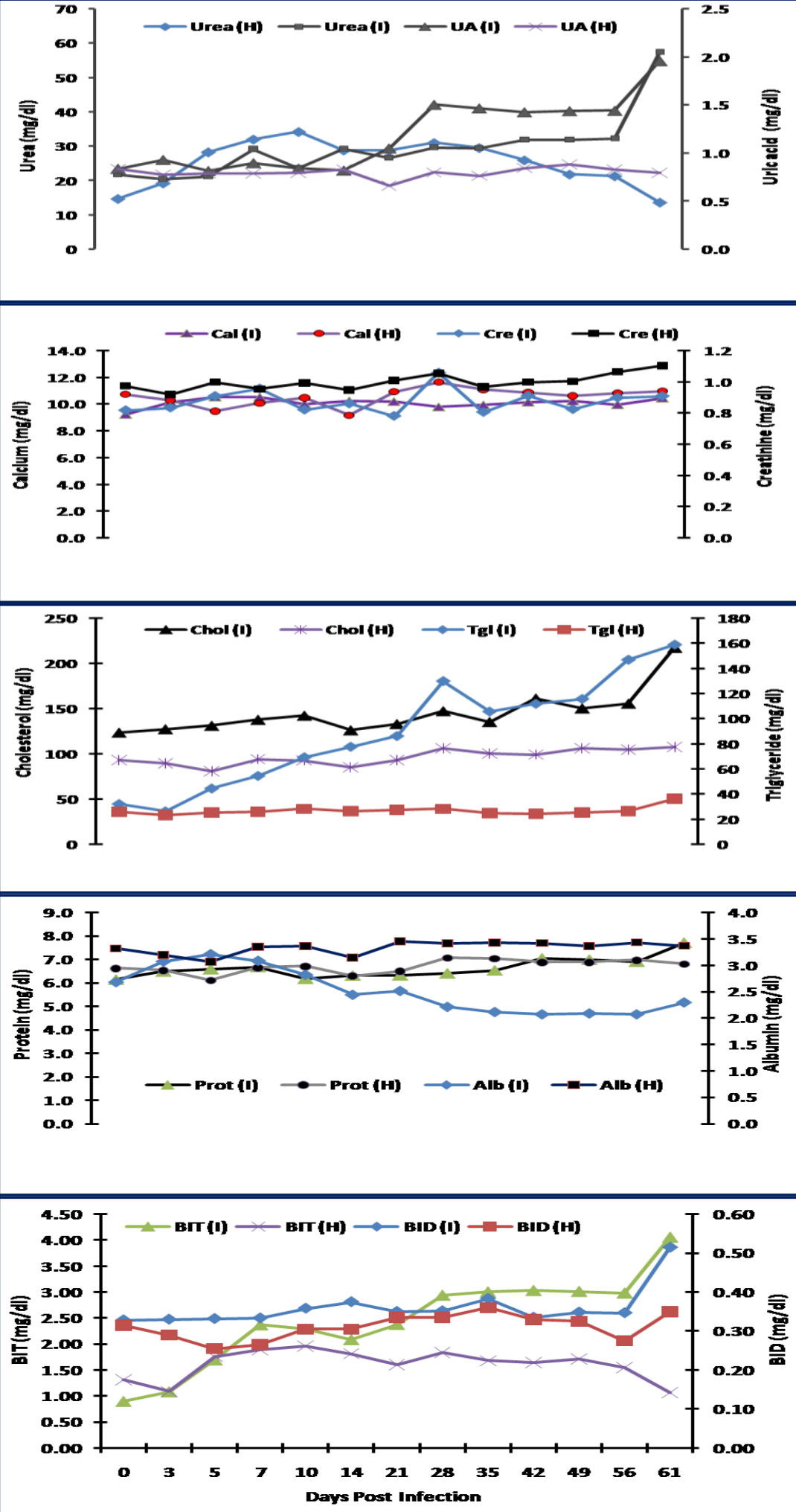

Figure 3 Changes in the level of various metabolites in $T$. evansi infected (I) and healthy (H) ponies during the study period. 


\section{Conclusion and Discussion}

In the present study, six healthy ponies inoculated subcutaneously with $T$. evansi, became positive within 5-7 DPI as detected by wet blood smear examination, however, prepatent period has been reported to vary from 4 to 13 days and parasitaemia displays an undulating course during the infection (Ramirez et al., 1979). In present investigation parasitaemia was consistent, throughout the experiment and all ponies developed acute disease within 56 days post-infection. Three ponies died exhibiting symptoms of acute trypanosomosis and fourth died due to neurological signs which appeared 10 weeks post-treatment with quinapyramine sulphate. Contrary to above observations, the experimentally infected donkeys showed comparatively low parasitaemia and the disease persisted years together in sub-clinical stage without mortality (Kumar et al., 2013).

These observations clearly indicated that ponies are susceptible of T. evansi infection, while donkeys are resistant with a long course of disease. The parasite count, body temperature and blood indices of the infected ponies underwent significant changes. The high temperature was observed in all infected ponies 8-15 DPI and was intermittent throughout the experimental period and there was no definitive correlation with peak parasitaemia. The increase in the temperature of infected animals is a characteristic that has been previously described and related to the waves of parasitaemia in experimental infections with T. evansi (Oshiro et al., 1989; Uche \& Jones 1992; Aquino et al., 1999; Marques et al., 2000; Dargantes et al., 2005). Thereafter, similar observations of intermittent fever, weakness, emaciation, anaemia, anorexia and incoordination in hind quarters were recorded as reported in experimental infection in equines (Marques et al., 2000; Wernery et al., 2001). Motorial disturbance, a frequent symptom of equine Surra, usually reported to affect mainly the hind legs (Curasson, 1943; Horchner et al., 1983) was also observed during this investigation.

The alterations in the haematological indices observed during the course of infection are consistent with the findings of previous workers in horses infected with T. evansi, wherein fall in haematocrit, erythrocyte counts and haemoglobin content was described. Anaemia has been a consistent finding in the infected animals throughout the study period. Marques et al. (2000) reported about 35\% decrease in red cells count, packed cell volume and haemoglobin concentration in the experimentally infected horses in three weeks of infection and thereafter small variations were observed. However, the animals remained anaemic until the end of observation period. Despite being a significant feature of trypanosomosis, the origin of anaemia in surra is not completely elucidated. Evidences suggest that its aetiology is multifactorial and haemolysis, haemodilution or/ and noncompensatory erythropoiesis are some of the mechanisms proposed (Jenkins \& Facer 1985). In conformity with our findings, death of $T$. evansi infected animals without clear previous indications has been also reported earlier (Horchner et al., 1983).
Among biochemical indices, both liver and kidney functions were observed to be affected in $T$ evansi infected ponies as their serum urea, uric acid, triglyceride, cholesterol, albumin, BID, BIT contents varied and increased significantly at different stages during experimental period. Similar observations mainly in terms of serum albumin and globulin have also been reported in donkeys (Cadioli et al., 2006).

\section{Acknowledgements}

The authors are thankful to Director, National Research Centre on Equines, Hisar for providing facilities to conduct the work. The authors also wish to acknowledge Department of Biotechnology (DBT), Government of India for partial funding through research Grant no. BT/PRi4499/ADV/57/107/2010 in terms of manpower support. The authors also wish to acknowledge and thank Mr. R.K. Dayal for technical support during the investigation.

\section{Conflict of interest statement}

All authors disclose that they have no financial and personal relationships with other people or organization that could inappropriately influence (bias) their work, including employment, consultancies, stock ownership, honoraria, paid expert testimony, patent applications/registration, and grants or other funding.

\section{References}

Ahmed A (2008) Epidemiological studies (parasitological, serological and molecular techniques) of T. evansi infection in camels in Egypt. Veterinary World Journal 1: 325-328.

Aquino LPCT, Machado RZ, Alessi AC, Marques LC, de Castro MB, Malheiros EB (1999) Clinical, parasitological and immunological aspects of experimental infection with Trypanosoma evansi in dogs. Memorias do Instituto Oswaldo Cruz 94: 255-260.

Aquino LPCT, Machado RZ, Alessi AC, Santana AE, Castro MB, Marques LC, Malheiros EB (2002) Hematological, biochemical and anatomopathological aspects of experimental infection with Trypanosoma evansi in dogs. Arquivo Brasileiro de Medicina Veterinária e Zootecnia 54: 8-18.

Berlin D, Loeb E, Baneth G (2009) Disseminated central nervous system disease caused by Trypanosoma evansi in a horse. Veterinary Parasitology 161: 316-319.

Cadioli FA, Marques LC, Machado RZ, Alessi AC, Aquino LPCT, Barnabé PA (2006) Experimental Trypanosoma evansi infection in donkeys: hematological, biochemical and histopathological changes. Arquivo Brasileiro de Medicina Veterinária e Zootecnia 58: 749-756. 
Curasson G (1943) Trypanosoma vivax et variétés. In Traité de protozoologie vétérinaire et comparée Tome 1 Trypanosomes. Paris: Vigot Frères : 270-278.

Dargantes AP, Reid SA, Copeman DB (2005) Experimental Trypanosoma evansi Infection in the Goat. I. Clinical Signs and Clinical Pathology. Journal of Comparative Pathology 133: $261-266$

Fernández D, González-Baradat B, Eleizalde M, GonzálezMarcano E, Perrone T, Mendoza M (2009) Trypanosoma evansi: A comparison of PCR and parasitological diagnostic tests in experimentally infected mice. Experimental Parasitology 121: 1-7.

Herrera HM, Alessi AC, Marques LC, Santana AE, Aquino LP, Menezes RF, Moraes MA, Machado RZ (2002) Trypanosoma evansi experimental infection in the South American coati (Nausa nausa): hematological, biochemical and histopatological changes. Acta Tropica 81: 203-210.

Hoare CA (1972) The Trypanosomes of Mammals: A Zoological Monograph. Blackwell Scientific Publications. Oxford, UK.

Horchner F, Schonefeld A, Wust B (1983) Experimental infection of horses with Trypanosoma evansi I. Parasitological and clinical results. Annales de la Societe Belge de Medecine Tropicale 63: 127-135.

Jenkins GC, Facer CA (1985) Hematology of African trypanosomes. In Tizard, I. Immunology and Pathogenesis of Trypanosomiasis. CRC Press, Boca Raton pp13-44.

Kumar R, Kumar S, Khurana SK, Yadav SC (2013) Development of an antibody-ELISA for seroprevalence of Trypanosoma evansi in equids of North and North-western regions of India. Veterinary Parasitology 196: 251-257.

Marques LC, Machado RZ, Alessi AC, Aquino LPCT, Pereira GT (2000) Experimental infection with Trypanosoma evansi in horses: clinical and haematological observations. Brazil Journal of Veterinary Parasitology 9: 11-15.
Oshiro ET, Rodrigues M, Nunes VLB, Ribeiro OC (1989). Trypanosoma (Trypanozoon) evansi (Steel, 1885) Balbiani, 1888, infecco experimental emequino com amostraisolada de capivara, Hydrochaeris hidrochaeris Linnaeus, 1766 (Rodentia: hydrochacridae). Semina 10: 51-55

Ramirez LE, Wells EA, Betancourt A (1979) La Tripanosomiases en los Animales Domisticos en Clolumbia. Centro Internacional de Agricultura Tropical pp 71.

Ranjithkumar M, Saravanan BC, Yadav SC, Kumar R, Singh R, Malik TA, Dey S (2013) Neurological trypanosomiasis in quinapyramine sulfate-treated horses - A breach of the bloodbrain barrier? Tropical Animal Health and Production 46: 371377.

Rodrigues A, Fighera A, Souza TM, Schild AL, Barros CSL (2009) Neuropathology of Naturally Occurring Trypanosoma evansi Infection of Horses. Veterinary Pathology 46: 251-258.

Soodan JS, Sood NK, Khahra SS (1996) Clinic-pathological studies in donkeys experimentally infected with Trypanosoma evansi. Indian Journal of Animal Science 66: 443-448.

Uche UE, Jones TW (1992) Pathology of experimental Trypanosoma evansi infection in rabbits. Journal of Comparative Pathology 106: 299-309.

Wernery U, Zachariah R, Mumford JA, Luckins T (2001) Preliminary evaluation of diagnostic tests using horses experimentally infected with Trypanosoma evansi. The Veterinary Journal 161: 287-300.

Woo PTK (1977) 7-Salivarian trypanosomes producing disease in livestock outside of sub-saharan Africa. In: Julius Kreir (Ed) Taxonomy Kinetoplastids and Flagellates of Fish. Academic Press, New York, pp 269-296.

Yadav SC, Kumar R, Manuja A, Goyal L, Gupta AK (2012) Early detection of Trypanosoma evansi infection and monitoring of antibody levels by ELISA following treatment. Journal of Parasitic Disease 38 : 124-127. 